\title{
Cloud \& Edge Trusted Virtualized Infrastructure Manager (VIM) - Security and Trust in OpenStack
}

\author{
Teodora Sechkova, Enrico Barberis, Michele Paolino \\ Virtual Open Systems \\ Grenoble, France \\ Email: \{teodora, e.barberis, m.paolino\}@virtualopensystems.com
}

\begin{abstract}
The Fifth Generation (5G) mobile networks promise faster connectivity and futuristic applications and services. In order to meet the high expectations, 5G joins forces with virtualization technologies like Network Functions Virtualization (NFV) and adopts cloud-native solutions. At the same time, it relies on shifting the computation to the network edge for offloading computing power, local caching, minimized latency and flexibility in the deployment. However, new opportunities unlock new security challenges. Man-in-the-middle, denial-ofservice attacks and tampering are now becoming easier because of the scattered devices and their varying locations. Meanwhile, the dynamic nature of the cloud raises the need for on-time threats prevention.

In this work, we propose a way to answer the new challenges by bringing trust into the virtualized edge infrastructure. We present our contributions to the development of security services for platform authentication and integrity, hosted inside a trusted execution environment (TEE). We also evaluate the performance overhead of our work and suggest future improvements.

Index Terms-security, virtualization, cloud, edge computing, Trusted Execution Environment, TEE, OP-TEE, ARM TrustZone, VIM, Virtual Infrastructure Manager, OpenStack
\end{abstract}

\section{INTRODUCTION}

The Fifth Generation (5G) mobile networks are promising greater data capacity and speed as well as enabling new applications and services. Cloud computing and virtualization have become an integral part of these networks that are today becoming more dynamic and agile thanks to cloud-native and edge computing technologies. In particular, edge computing brings the benefits of reduced network traffic, locationawareness and low-latency while cloud-native solutions improve performance and reduce cloud services footprint. However, both open new security challenges. In fact, the edge computing scattered architecture and the wireless connectivity increase the risk of man-in-the-middle attacks, with a major threat of device tampering or replacement. Moreover, cloudnative solutions aim to simplify all levels of the $5 \mathrm{G}$ software stack, optimizing it for cloud execution and removing unnecessary dependencies with bare metal solutions. Nevertheless, it significantly relies on the virtualization infrastructure for security. Among the many known aspects of the cloud-native and edge security [1] [2], the protection of the virtualization infrastructure and the platform integrity are the focus of this work.

We propose a trusted edge computing infrastructure based on Arm edge devices. In addition, we add attestation exten- sions to OpenStack [3] [4], one of the most widely adopted open-source cloud managers. The combination of a remote attestation integrated into the infrastructure management software and a trusted pool of devices brings a secure and trusted virtualized edge.

As part of this work, we perform a series of measurements showing the trade-off between performance and increased security. The experiments are conducted using standard virtual machines (VMs), showing the worst-case scenario in terms of instantiation (boot) times, however, the same concept is still applicable when working with unikernels or containers.

The next sections are organized as follows. Section II presents a background in the trusted computing area. The architecture, as well as details of the implementation of the proposed solution, are described in Section III. The experimental setup and result can be found in Section IV, followed by the related work and the state-of-the-art in Section V. The authors' conclusions and an outline of future efforts are expressed in Section VI.

\section{TRUSTED COMPUTING BACKGROUND}

Building a secure cloud and edge infrastructure requires running services and workloads onto trusted hosts. This is an important part of the resource placement process in any cloud and edge management platform. Different properties and principles have been identified in order to achieve this task [5], [6]. They can be summarized as the need for a hardware root of trust on devices and the possibility of an external attestation. Two standardization bodies are leading the efforts into defining a common way of applying trusted computing technologies. The Trusted Computing Group (TCG) has defined the Trusted Platform Module (TPM) [7] and The Global Platform released the Trusted Execution Environment (TEE) specification [8]. The two technologies can be seen as competitive but there are also efforts towards their convergence since the TPM requirements can be implemented not only in hardware but also in software inside a TEE [9]. Worth to mention hardware technologies that are based on these specifications are outlined next.

- Intel Trusted Execution Technology (TXT) [10] aims to provide a root of trust and verify the integrity of a platform by relying on a TPM. During boot, it performs measurements on the platform components (boot loader, 
firmware, hypervisor, operating system) and verifies them against pre-calculated white list values.

- Intel SGX or Intel Software Guard Extensions [11] is an extension for Intel processors architecture which enables the use of protected areas of execution in memory, called enclaves. It makes an application code executing within the enclave protected even when the firmware or operating system are compromised.

- Arm TrustZone [12] implements a TEE as a system-wide approach to security. TrustZone is hardware-based security built into System-on-Chips (SoCs) by semiconductor chip designers who want to provide secure end-points and a device root of trust.

Arm devices historically have become of common usage in resource-constrained environments. Such constraints are also valid in the world of $5 \mathrm{G}$ and edge computing and for these reasons we are focusing our efforts on this type of hardware.

The TrustZone technology is available in older Arm architectures but the highest Exception Level (EL3) is introduced with ARMv8. The EL3 manages the context switch between a Secure and a Normal world by hardware exceptions and is considered secure irrespective of the state of the Central Processing Unit (CPU). Relying on this strong isolation, the Secure world can be used to propagate a chain of trust during system boot. Other sensitive operations such as key storage and cryptography tasks are also commonly performed inside the Secure World [13].

In addition to the hardware technologies, a number of software implementations of the Global Platform TEE exist. The commercial works are not of interest to this paper, while a short overview of the open-source ones is presented here. Open Portable Trusted Execution Environment (OP-TEE) [14] meets the GlobalPlatform TEE System Architecture specification and provides the TEE Internal Core API and TEE Client API. Other available implementations include Open TEE led by the Secure Systems group as part of their activities at the Intel Collaborative Research Institute for Secure Computing [15] and T6 which targets mobile devices using Arm hardware security extensions [16].

The work on the hardware isolation and trust needs to be complemented by attestation capabilities. The authors in [6] give a formal definition of the attestation process as the activity of making a claim to an appraiser about the properties of a target by supplying evidence which supports that claim. Among the many properties of a device, the authenticity and the integrity of the software stack are the most critical ones when it comes to security and are the focus of the authors.

\section{TRUstedVIM}

The proposed solution in this work is called Trusted Virtualized Infrastructure Manager (TrustedVIM). It supplements the management capabilities of OpenStack with attestation extensions integrated directly into the OpenStack Compute (Nova) project. The key component is the compute infrastructure which is based on Arm TrustZone enabled hosts and OP-TEE where all the security services are isolated and safely executed inside a TEE. Relying on this framework, OpenStack compute nodes are being authenticated before cloud services such as virtual machines (VMs), unikernels or potentially containers [17] can be run on them, this way avoiding manin-the-middle or device replacement attacks. Furthermore, the operating system kernel is checked for unknown modifications, detecting malicious activities and a trusted boot ensures that the trustworthiness of the system is propagated throughout all software layers.

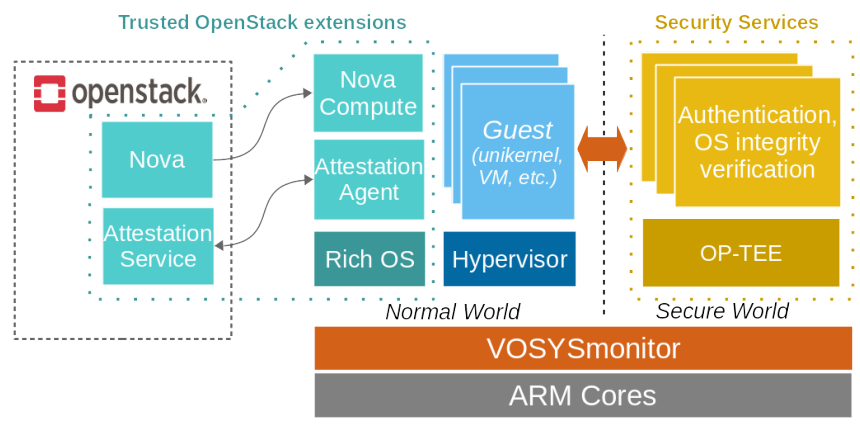

Fig. 1. TrustedVIM architecture

\section{A. Architecture}

TrustedVIM architecture is shown in Figure 1. It consists of two components: Trusted OpenStack extensions and OPTEE Security services. Trusted OpenStack extensions add an attestation service and an attestation agent to the existing OpenStack environment. They are part of the instance (VM) scheduling process and are responsible for the verification of the nodes' authenticity and integrity. On the request of a new OpenStack instance creation, the attestation service filters the available compute nodes by their trust state. Only nodes that are decided to be trustworthy are passed through and can be used as hosts for the instance placement.

The base for the compute nodes trust verification is the security services running on the hosts. The trusted compute infrastructure is made of Arm TrustZone-enabled nodes running VOSYSMonitor [18]. This is a thin monitoring software layer developed by Virtual Open System which manages the interaction between two execution worlds on a single Systemon-Chip.

This mixed-critical infrastructure provides each node with an isolated environment for storing and running sensitive data and applications. Each host has two execution environments, called a Normal World and a Secure World. The Normal World hosts a rich operating system, Linux in the case of this work, where the nova-compute services and the guest VMs are running. Meanwhile, the Secure World works with OP-TEE to provide a secure environment for the critical tasks of the Normal World. The Security Services are OP-TEE applications and are the trusted source for the device authenticity and integrity. 


\section{B. OP-TEE Security services}

Verifying that a compute node is not compromised is a challenging goal. However, some mechanisms can be put in place to detect infection symptoms. Having in mind this problem, the following services are implemented to take countermeasures:

- Secure boot is a security mechanism applied to verify the trustworthiness of the software during each stage of the device boot process. It is implemented as a chain of trust starting from a hardware root of trust and propagates up to the user-space.

- Node authentication is performed before starting the communication with a compute node. The OpenStack Controller uses a "Challenge-response" protocol, based on asymmetric cryptography, to verify that the compute node is actually the one that it is claiming to be. This is a fundamental service to avoid man-in-the-middle attacks.

- Kernel integrity check: in order to interfere with the execution of a VM a malicious software (commonly referred to as malware) has to gain high privileges. Usually, an infection of the kernel space is needed. The main malware category that aims at this are the so-called "Rootkits". One common technique used by rootkits is to tamper the kernel code to perform malicious actions. A valid countermeasure is a run-time integrity check of the kernel code memory. Although this doesn't prevent the execution of a Rootkit but only the detection of it, this information is valuable for the OpenStack Controller that can mark the infected node as not trusted and take countermeasures.

These services can be implemented in normal user space but this will be insecure. In order to explain it, we model an attack as a generic rootkit. Rootkits can tamper any application in the user and kernel space including the above security services. Here comes in help the hardware isolation provided by Arm TrustZone. By implementing the security services as OP-TEE applications inside the Secure world, they cannot be affected by software running in the Normal World and are protected from malware and rootkits. Meanwhile, through the Global Platform Client API a client software running in the Rich OS can ask OP-TEE for the safe execution of these services.

\section{Implementation details}

In this subsection, the different parts of the TrustedVIM architecture are explained in terms of their implementation. The Trusted OpenStack extensions are implemented as part of the Nova project with more details following. Together with the OP-TEE security services, they form the two main features of our work, namely, host authentication and kernel integrity check.

1) Trusted Boot: A system's security cannot be guaranteed if it does not start from the moment of power-on. The trusted boot is the basis for the trustworthiness of the upper software layers in this work. It is accomplished by the vendor-specific secure boot mechanism relying on a hardware root of trust and is propagated to the Linux user-space by U-Boot's verified boot [19]. U-boot is an open-source boot loader available for multiple computer architectures (Arm, x86, etc.). The boot process is multi-staged and starts from the boot read-only memory (ROM) and the first-stage boot loader (FSBL). After the FSBL is determined as secure, it loads U-Boot which on its turn verifies the signature of the Linux kernel image and only then loads the operating system.

2) Attestation service: Nova-scheduler is one of the internal components of OpenStack Nova and is part of the flow for provisioning a new VM instance. By default, the novascheduler is configured as a filter scheduler. Filtering is a mechanism inside Nova for making decisions about where a new instance should be created. During this process the scheduler iterates through all compute nodes, evaluating each against a set of filters. The list of resulting hosts is ordered by weights and is used to select the one that meets all VM requirements. If the scheduler cannot find candidates, it means that there are no appropriate hosts where that instance can be scheduled [20].

We implemented a custom filter, called attestation_filter, running inside the controller, together with an attestation_agent application running on each compute node. The attestation filter is run during the default filtering procedure of the scheduler. It establishes a connection with the attestation_agent and gathers information about the trust status of the platform. Based on this data it informs the scheduler if each of the hosts passes or not.

The attestation process is activated by adding an additional property "trusted" to any of the OpenStack flavors [21]. If no metadata is added OpenStack works as in its vanilla deployment.

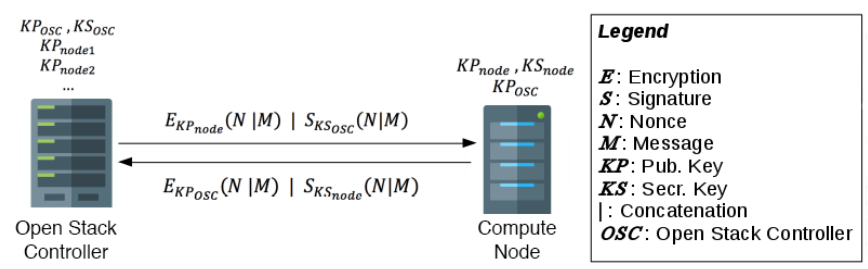

Fig. 2. TrustedVIM challenge-response protocol

3) Node authentication: The authentication of a node is a delicate problem. It is important to correctly use the available cryptographic tools to avoid possible attacks and define a secure communication channel. For the node authentication service the following considerations can be made:

- Authenticity: this, as expected, is the main goal. To obtain this result the use of Asymmetric cryptography is mandatory.

- Integrity: at first glance, these requirements may seem not mandatory but it is as important as authenticity. Indeed, in case of man-in-the-middle attacks, we want to be sure that any message tampering is detected and signalled to the OpenStack Controller. In this way, every message is not only authentic but also valid. 
- Secrecy: keeping secret the exchanged messages is not mandatory but can be a plus to avoid leaking of information.

Having in mind these three goals, the protocol implemented is shown in Figure 2.

The OpenStack Controller knows the public key of all the compute nodes, while all the compute nodes know the public key of the OpenStack Controller. Obviously, the private key is known only by the actor itself. Thanks to this, it is possible to exchange a packet composed of the following elements:

- The encrypted message: thanks to this secrecy is granted since only the receiver can decrypt the content of the message. Moreover, the presence of a nonce makes ineffective reply attacks.

- The signature of the message: this acts as a Message authentication code (MAC). It guarantees the authenticity and integrity of the packet

By following this protocol, we achieve a secure communication secure channel that allows end-to-end encryption between the OpenStack Controller and the OP-TEE operating system.

Figure 3 shows how the protocol is implemented on a compute node. Even if the Linux kernel on the compute node is comprised, an attacker cannot alter this communication channel. The only possible attack is the interruption of the channel, but this can be already interpreted as a "red flag" for the OpenStack Controller.

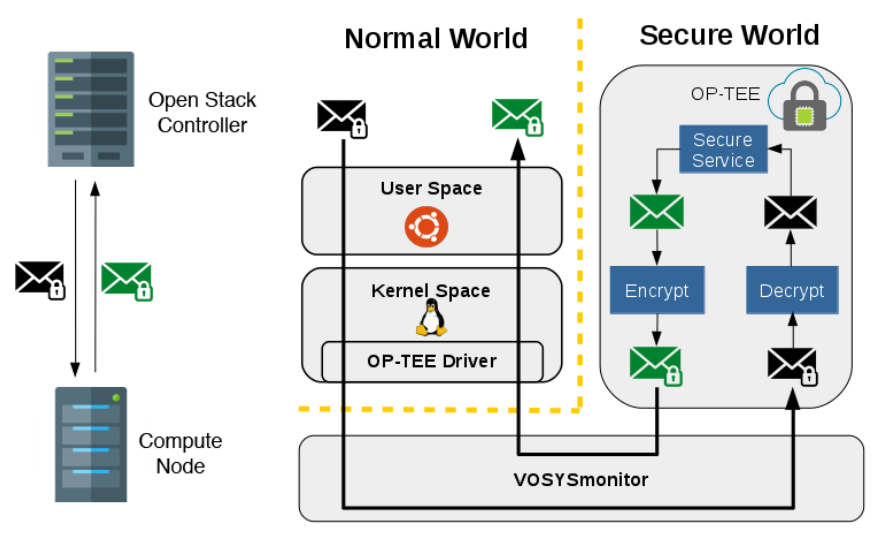

Fig. 3. End-to-end communication diagram

4) Kernel integrity check: Detecting all signs of infection is almost an unachievable task. Having said that, this implementation intends to serve as a proof of concept and to demonstrate the feasibility of the work and measure its performance. We use Rootkit as the main attack vector and implement the Linux integrity check by monitoring two essential tables present in the Linux kernel:

- sys_call_table: this table contains all the pointers to the functions implementing the system calls. Since the interface between user and kernel space goes entirely through this interface, it is mandatory to verify the integrity of this table to avoid tampering of those.

- vectors: in the Arm architecture whenever an exception occurs the processor switches the execution to the correct entry in the vector table. This table is also critical for safety and it should not be never changed.

Thanks to OP-TEE it is possible to verify at run-time that these tables content is not modified. To perform this it is sufficient to compute the digest of these tables and verify that it is exactly as the one expected. OP-TEE embeds in its code the digests of the tables computed offline using the verified kernel image. Figure 4 summarizes the steps of this integrity check.

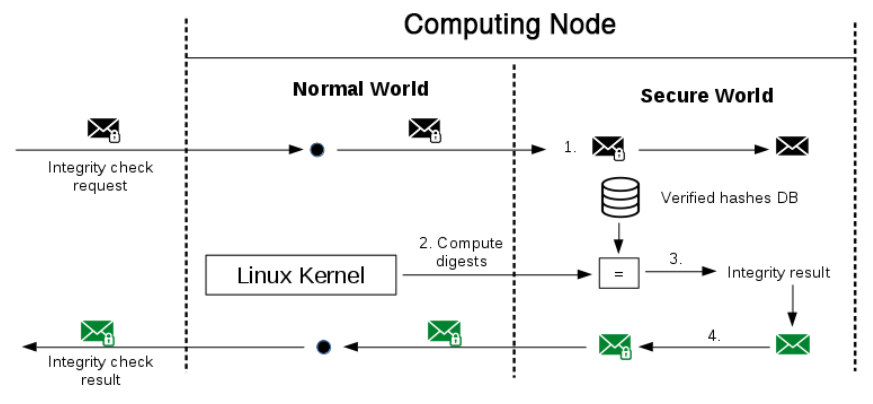

Fig. 4. Integrity check diagram

\section{EXPERIMENTAL SETUP AND RESULTS}

In order to evaluate the overhead added by the newly implemented security features, an experiment is designed to compare the same OpenStack deployment with and without added trust. The implementation done in this paper directly affects the VM creation process and the experimental set-up is created accordingly. It measures and compares the boot and delete times of a VM in two scenarios: with and without trust features enabled. The OpenStack project Rally [22] is used to conduct the experiment. This is an open-source generic testing tool for OpenStack multi-node deployment evaluation [23].

OpenStack is deployed with Devstack [24] in a multi-node configuration:

- One x86 controller node: Intel(R) Xeon(R) CPU E5-2623 v4 @ 2.60GHz, 32GB memory, Ubuntu 16.04.4 LTS, KVM-enabled 4.4.0-128-generic Linux kernel

- One aarch64 compute node: Xilinx Zynq UltraScale+ MPSoC ZCU102 with a quad-core ARM Cortex-A53, 4GB memory, Ubuntu 18.04.4 LTS, KVM-enabled 4.14.0-dirty Linux kernel

The hardware configuration of the virtual machines is based on the OpenStack m1.tiny flavor (1 VCPU, 1GB Disk, 512MB RAM) and the booted guest OS is a CirrOS cloud image. The experiment is conducted by consecutively booting and deleting $50 \mathrm{VMs}$. In its first part, in order to measure the vanilla OpenStack timing, we use default m1.tiny flavor. In the second part, the same flavor is extended with a new property "trusted" to enforce the trust requirements.

The results are shown in Table I and Table II. The atomic operations used by Rally for booting and deleting a VM are called nova.boot_instance and nova.delete_instance respectively. 
TABLE I

BOOT AND DELETE 50 VMS, WITHOUT TRUSTED FEATURES

\begin{tabular}{lccccccc}
\hline Action & Min $(\mathbf{s e c})$ & Median(sec) & $\mathbf{9 0 \% \text { ile(sec) }}$ & 95\%ile(sec) & Max(sec) & Avg(sec) & Count \\
\hline nova.boot_instance & 12.816 & 12.939 & 13.084 & 13.152 & 13.4 & 12.97 & 50 \\
\hline nova.delete_instance & 2.35 & 2.366 & 2.39 & 2.5 & 2.55 & 2.378 & 50 \\
\hline total & $\mathbf{1 5 . 1 8 2}$ & $\mathbf{1 5 . 3 1 9}$ & $\mathbf{1 5 . 5 0 4}$ & $\mathbf{1 5 . 5 5 4}$ & $\mathbf{1 5 . 7 7 8}$ & $\mathbf{1 5 . 3 8 4}$ & $\mathbf{5 0}$ \\
\hline
\end{tabular}

TABLE II

BOOT AND DELETE 50 VMS, WITH TRUSTED FEATURES

\begin{tabular}{lccccccc}
\hline Action & Min $(\mathbf{s e c})$ & Median(sec) & $\mathbf{9 0 \% \text { ile(sec) }}$ & $\mathbf{9 5 \% \text { ile(sec) }}$ & Max(sec) & Avg(sec) & Count \\
\hline nova.boot_instance & 12.844 & 12.986 & 13.324 & 15.186 & 17.216 & 13.223 & 50 \\
\hline nova.delete_instance & 2.356 & 2.371 & 2.395 & 2.569 & 2.62 & 2.388 & 50 \\
\hline total & $\mathbf{1 5 . 2 1}$ & $\mathbf{1 5 . 3 8 6}$ & $\mathbf{1 5 . 8 3 3}$ & $\mathbf{1 7 . 5 5 8}$ & $\mathbf{1 9 . 7 9 4}$ & $\mathbf{1 5 . 6 1 1}$ & $\mathbf{5 0}$ \\
\hline
\end{tabular}

As expected the delete times are not affected by the security features. The TrustedVIM plays a part only during the VM scheduling process. The trusted implementation is 0.253 seconds $(\sim 2 \%)$ slower on average but looking at the detailed statistics, it can be seen that the minimum and median values are only slightly affected. The main difference comes from peaks into the measurements visible in the graphical representation on Figure 6 . This can be explained by fluctuations of the connectivity speed and is a basis for future improvement.

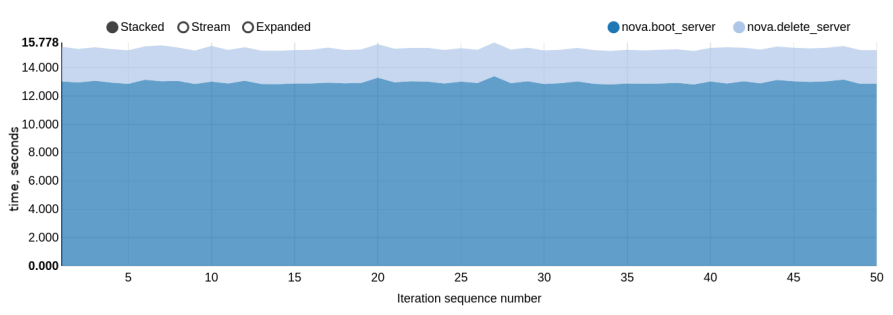

Fig. 5. VM boot and delete times (sec), without trusted features

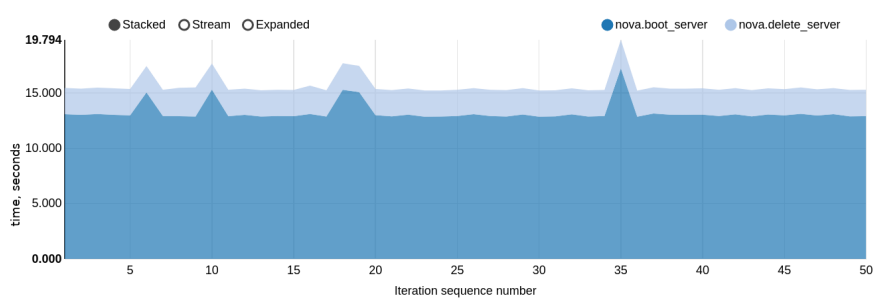

Fig. 6. VM boot and delete times (sec), with trusted features

\section{RELATED WORK}

The topic of trusted computing pools is being explored by Intel in a combination with the OpenAttestation project (OAT)
[25] and OpenStack. Their work is based on Intel TXT which creates a Measured Launch environment (MLE) on power-on [10]. Intel TXT relies on a TPM as a hardware root of trust and for storing the measurements. Compute nodes based on Intel TXT are combined with a remote attestation software to provide Trusted computing pools to OpenStack. During an instance scheduling, the host selection process is extended with additional attestation (performed by an external OAT server) which verifies that the compute nodes are launched correctly and can be trusted. The support for this functionality is no longer continued in the latest releases of OpenStack due to lack of tests and completeness [26].

Another Intel based solution is proposed by [27]. This time the HW technology used is Intel SGX. The authors reflect on the security guarantees of containers and propose an SGX-aware architecture for orchestrating containers based on Kubernetes [28]. The system runs on a heterogeneous cluster and is able to efficiently schedule regular and SGX-aware jobs.

In the work of [29], a security architecture for ARMv8 is proposed, called T-KVM (Trusted Kernel-based Virtual Machine). It offers a strong isolation for guest operating systems by means of the KVM hypervisor, Arm TrustZone, SELinux and a virtualization enabled implementation of the GlobalPlatform TEE API. Part of it is based on open-source components like KVM and libvirt [30] which eases a potential integration with OpenStack.

As expected from the definition of the problem itself, all of the presented works are tightly coupled with hardware technologies. [10] and [27] are adding additional value through the combination with an orchestration and management software, basing their work on Intel chips which are dominating the cloud data center. Meanwhile, [29] focus on Arm and opensource components but leave the potential integration with OpenStack as future efforts. 


\section{CONCLUSION}

In this work, a new virtualization-based security and trust infrastructure for Arm edge devices is proposed. It complements a well-known VIM with attestation features and adds security services inside a trusted hardware-isolated environment in order to meet some of the NFV and 5G security challenges. This is a proof of concept for hardening the security of the infrastructure against man-in-the-middle, denial-of-service or device tampering attacks.

In addition, we evaluate the computing overhead associated with the trusted features and show that it is an acceptable penalty against its security benefits. Moreover, future efforts for improving the implementation of the attestation process can reduce this overhead even further.

There are multiple opportunities for a future expansion of the work with new features like asset and geo-tagging, aiming at the goal of workload location control and management. Another big topic of research is the rivalry between VMs and containers on the edge. The light-weight nature of unikernels and containers makes them suitable for the resourceconstrained edge and the future extension for their support and a comparison with the current results is of interest to the authors.

\section{ACKNOWLEDGMENT}

This work has received funding from the European Union's Horizon 2020 research and innovation programme under grant agreement No 761508 (H2020 5GCity project).

\section{REFERENCES}

[1] S. Lal, T. Taleb, and A. Dutta, "Nfv: Security threats and best practices," IEEE Communications Magazine, vol. 55, no. 8, pp. 211-217, 2017.

[2] R. Roman, J. Lopez, and M. Mambo, "Mobile edge computing, fog et al.: A survey and analysis of security threats and challenges," Future Generation Computer Systems, vol. 78, pp. 680-698, 2018.

[3] "Openstack," [accessed 23-January-2019]. [Online]. Available: https://www.openstack.org/

[4] T. Sechkova, M. Paolino, and D. Raho, "Virtualized infrastructure managers for edge computing: Openvim and openstack comparison," in 2018 IEEE International Symposium on Broadband Multimedia Systems and Broadcasting (BMSB). IEEE, 2018, pp. 1-6.

[5] G. Hunt, G. Letey, and E. Nightingale, "The seven properties of highly secure devices," tech. report MSR-TR-2017-16, 2017.

[6] G. Coker, J. Guttman, P. Loscocco, A. Herzog, J. Millen, B. OHanlon, J. Ramsdell, A. Segall, J. Sheehy, and B. Sniffen, "Principles of remote attestation," International Journal of Information Security, vol. 10, no. 2, pp. 63-81, 2011.

[7] "Tpm main specification," [accessed 23-January-2019]. [Online]. Available: https://trustedcomputinggroup.org/resource/tpm-mainspecification/

[8] "Global platform specifications," [accessed 23-January-2019]. [Online]. Available: https://globalplatform.org/specs-library/

[9] H. Raj, S. Saroiu, A. Wolman, R. Aigner, J. Cox, P. England, C. Fenner, K. Kinshumann, J. Loeser, D. Mattoon et al., "ftpm: A firmware-based tpm 2.0 implementation," Microsoft Research, 2015.

[10] J. Greene, "Intel trusted execution technology (white paper)," Online: http://www. intel. com/txt, 2012.

[11] V. Costan and S. Devadas, "Intel sgx explained." IACR Cryptology ePrint Archive, vol. 2016, no. 086, pp. 1-118, 2016.

[12] T. Alves, "Trustzone: Integrated hardware and software security," White paper, 2004.

[13] P. Lucas, K. Chappuis, M. Paolino, N. Dagieu, and D. Raho, "Vosysmonitor, a low latency monitor layer for mixed-criticality systems on armv8-a," in LIPIcs-Leibniz International Proceedings in Informatics, vol. 76. Schloss Dagstuhl-Leibniz-Zentrum fuer Informatik, 2017.
[14] "Open portable trusted execution environment," [accessed 23-January2019]. [Online]. Available: https://www.op-tee.org/

[15] "Open tee," [accessed 23-January-2019]. [Online]. Available: https://open-tee.github.io/

[16] L. Wen-Hao, "T6, an operating system for trustzone based trusted execution environment (tee) in arm-based systems," White paper, 2014.

[17] "Leveraging containers and openstack," [accessed 23-January-2019]. [Online]. Available: https://www.openstack.org/containers/leveragingcontainers-and-openstack/

[18] P. Lucas, K. Chappuis, B. Boutin, J. Vetter, and D. Raho, "Vosysmonitor, a trustzone-based hypervisor for iso 26262 mixed-critical system," in 2018 23rd Conference of Open Innovations Association (FRUCT). IEEE, 2018, pp. 231-238.

[19] S. Glass, "Verified u-boot," 2013.

[20] "Openstack schedulung," [accessed 23-January-2019]. [Online]. Available: https://docs.openstack.org/mitaka/configreference/compute/scheduler.html

[21] "Openstack flavors," [accessed 23-January-2019]. [Online]. Available: https://docs.openstack.org/horizon/latest/admin/manage-flavors.htmln

[22] "Rally documentation," [accessed 23-January-2019]. [Online]. Available: https://rally.readthedocs.io/en/latest/

[23] T. Pflanzner, R. Tornyai, B. Gibizer, A. Schmidt, and A. Kertesz, "Performance analysis of an openstack private cloud," 2016.

[24] "Devstack documentation," [accessed 23-January-2019]. [Online]. Available: https://docs.openstack.org/devstack/latest/

[25] "Openattestation project," [accessed 23-January-2019]. [Online]. Available: https://01.org/OpenAttestation

[26] “Openstack documentation," [accessed 23-January-2019]. [Online]. Available: https://docs.openstack.org/nova/pike/user/filter-scheduler.html

[27] S. Vaucher, R. Pires, P. Felber, M. Pasin, V. Schiavoni, and C. Fetzer, "Sgx-aware container orchestration for heterogeneous clusters," arXiv preprint arXiv:1805.05847, 2018.

[28] J. Ellingwood, "An introduction to kubernetes," Retrieved April, vol. 15, p. 2017, 2016.

[29] M. Paolino, A. Rigo, A. Spyridakis, J. Fanguède, P. Lalov, and D. Raho, "T-kvm: A trusted architecture for kvm arm v7 and v8 virtual machines securing virtual machines by means of kvm, trustzone, tee and selinux."

[30] R. Hat, "libvirt: The virtualization api," 2012. 